\title{
Impacto biomédico de los viajes en adultos mayores chilenos
}

\author{
Pedro Paulo Marín L, Marcela Carrasco G, \\ Mauricio Cabezas Ua, Homero Gac E, Trinidad Hoyl M, \\ Patricio Duery Da, Kristina Petersen Ca, \\ Catalina D ussaillant $\mathbf{K}^{\mathbf{b}}$.
}

\section{Biomedical impact of traveling for Chilean elderly}

Background: In Chile there is a program named "Vacations for Elderly during Low Season". Aim: To characterize participants of this program and to measure the impact of traveling in their health and wellbeing. Material and methods: Two anonymous and voluntary questionnaires were applied to 4200 participants, before and after a ten days vacation package. Results: Before traveling, questionnaires were answered by 802 subjects, and after traveling by 4057 (69\% women, 22\% older than 75 years old, 15.8\% living alone). The presence and maintaining of good health were most appreciated at this age and $59 \%$ classified their health as good or excellent. Twenty five percent referred sensory problems (seeing or hearing), $12 \%$ reported urinary incontinence and $21 \%$ presented falls in the last three months; depression screening (GDS5) was positive in 16\%. Chronic disease prevalence was similar to the general Chilean elderly population. After traveling they reported significant improvements in the items sociability, wellbeing, mood, appetite, insomnia and ostheoarthritic pain. Conclusions: Elderly who traveled were mainly women, who thought that maintaining good health is the most precious value. After traveling they improved significantly different aspects of wellbeing. Promotion of this kind of recreation programs is an important tool for integration and enhancement of quality of life in elderly subjects in our country (Rev Méd Chile 2004; 132: 573-8).

(Key Words: Aged; Fail elderly; Travel)

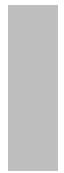

Recibido el 17 de noviembre, 2003. Aceptado en versión corregida el 30 de marzo, 2004. Programa de Geriatría y Gerontología, Departamento de Medicina Interna, Facultad de Medicina, Pontificia Universidad Católica de Chile.

anternos de Medicina, Facultad de Medicina, Pontificia Universidad Católica de Chile. bAlumna de Medicina, Facultad de Medicina, Pontificia Universidad Católica de Chile.

Correspondencia a: Dr. Pedro Pablo Marín L Programa de Geriatría y Gerontología, Departamento de Medicina Interna, Facultad de Medicina, Pontificia Universidad Católica de Chile. Casilla 114-D, Santiago, Chile. E-mail: ppmarin@med.puc.cl 
$\mathrm{C}$ hile está envejeciendo: según el censo 2002 los adultos mayores de sesenta años (adultos mayores, $\mathrm{AM}$ ) son $11,4 \%$ de la población total $\mathrm{y}$, mientras la población general del país creció a tasas de 2,1\% anual, los AM lo hicieron a un ritmo de $3,3 \%{ }^{1}$. Es por esto que el desafío del envejecimiento cobra mayor importancia político-social y debemos prepararnos como país para enfrentar$\mathrm{lo}^{2,3}$.

En Viena (1982), se realizó la primera "Asamblea Mundial sobre Envejecimiento", donde se aprobó el Plan de Acción Internacional para la elaboración de políticas sobre envejecimiento. Recientemente, en Madrid (2002), se realizó la segunda asamblea y se reformuló este plan de acción, destacándose la necesidad de "cambios en las actitudes, las políticas y las prácticas a todos los niveles y en todos los sectores, para que puedan concretarse las enormes posibilidades que brinda el envejecimiento del siglo XXI". Dicho plan consta de sesenta y dos recomendaciones en siete ámbitos generales, una de las cuales es el bienestar social, que incluye incentivar la recreación y el mejor aprovechamiento del ocio en los adultos mayores ${ }^{4}$.

Paralelo a la asamblea de Madrid se realizó un foro sobre "Viajes y Tercera Edad", donde se presentaron las experiencias de diferentes países y se abordaron los beneficios de dichos programas, cómo sacar a los AM del aislamiento, luego de jubilarse; devolverles el placer de desplazarse para preservar el mayor tiempo posible tanto su independencia física como social; integrarlos en una actividad grupal a fin de que renueven el contacto con los demás; aumentar la capacidad de expresarse, comunicarse y participar; brindarles la posibilidad de visitar distintos lugares y ofrecerles mayor autonomía, como también hábitos de conducta saludables. La presencia internacional y especialmente latinoamericana en ese foro estimuló a la asociación de agentes en esta apuesta por hacer de la recreación un componente estratégico de las políticas públicas sociales de nuestros países. Se demostró que los servicios orientados a este grupo de edad han logrado una alta efectividad en la estimulación afectiva de las personas mayores, potenciando sus posibilidades de integración social. Se concluyó que se debe "evitar la segmentación de la vida del ser humano en espacios sucesivos de aprendizaje, trabajo y final- mente ocio. Se solicita que estos aspectos (aprendizaje, trabajo y descanso) se integren de manera simultánea durante toda la vida y se impulse el manejo constructivo y creativo del ocio durante toda la vida” ${ }^{\prime \prime}$.

La idea de promover un programa subvencionado de vacaciones para el AM surgió en 1985, en España, con dos principales aspiraciones: mejorar la calidad de vida de los AM y favorecer el mantenimiento del empleo en baja temporada. Estos principios fueron analizados y ratificados posteriormente en los foros sobre Turismo y Tercera Edad de Brasil (1996) y de Portugal (1999). En España (1989), el Instituto Nacional de Servicios Sociales (INSERSO) confirmó el efecto positivo macroeconómico del "Programa Vacaciones de Tercera Edad". En Chile, esta iniciativa comenzó el año 2001, cuando el Servicio Nacional de Turismo (SERNATUR) coordinó el Programa "Vacaciones de Tercera Edad en Baja Temporada".

El presente estudio descriptivo, pretende conocer el perfil del AM usuario de este programa en Chile y evaluar el impacto biomédico de los viajes en nuestros AM. No encontramos en la literatura información a este respecto, sólo hay algunos datos sobre evaluación de calidad y satisfacción con los servicios recibidos ${ }^{9-10}$.

\section{MATERIAL Y MÉTODO}

En 2001, SERNATUR comenzó en nuestro país el programa de "Vacaciones de Tercera Edad en Baja Temporada", que fue licitado y adjudicado a la empresa IberoJet. Ese año el Centro de Geriatría y Gerontología de la Pontificia Universidad Católica de Chile firmó un convenio con dicha empresa, para estudiar, mediante una encuesta, el impacto biomédico-social de los AM que viajarían en estos programas. Este estudio, además, contó con la aprobación del Director de SERNATUR.

La encuesta de evaluación e impacto fue diseñada por nuestro grupo y sometida a la crítica de especialistas extranjeros, siendo la versión final acordada con expertos de la OPS-OMS. A todos los que viajaron durante el primer trimestre de 2002 $(n=4.200)$, en forma prospectiva y no seleccionada, se les entregaron dos encuestas, cuya respuesta era voluntaria y anónima, para ser respondidas el primer día y al regresar desde uno de los cinco 
destinos (Viña del Mar, Valdivia, La Serena, Iquique y Arica) donde permanecieron diez noches.

La encuesta para contestar el primer día, PREVIAJE, contenía cinco bloques: 1) datos demográficos generales, 2) evaluación sobre priorización de temores y valores más relevantes en esta etapa de la vida; 3) Evaluación geriátrica, que incluía: autopercepción de salud, déficit sensoriales, uso de medicamentos, evaluación funcional, tamizaje de depresión (Escala de Depresión Geriátrica acortada GDS-5) ${ }^{6}$, evaluación social (estimación de recursos autoevaluando estado económico) y evaluación de red de apoyo; 4) Tamizaje sobre problemas geriátricos prevalentes (insomnio, tabaquismo, caídas e incontinencia urinaria); 5) Antecedente de patologías conocidas (osteoporosis, hipertensión arterial, diabetes mellitus, enfermedad coronaria, enfermedad pulmonar crónica, y otras).

La encuesta POSTVIAJE era mucho más corta y evaluó de qué forma influyó el viaje en los siguientes aspectos: molestias articulares, ánimo, apetito, sueño, digestión, memoria, bienestar general, sociabilidad; solicitándose marcar entre las siguientes categorías: Peor, Un poco peor, Sin cambios, Mejor o Mucho mejor.

Para el análisis estadístico se utilizó el programa SPSS; para la comparación de promedios se usó el test de $t$ de Student y para la comparación de porcentajes el test de Chi cuadrado. Se consideró significativo un valor $\mathrm{p}<0,05$.

\section{RESULTADOS}

La población analizada en este estudio descriptivo, prospectivo y no seleccionado, fueron aquellos AM que contestaron completamente alguna de las dos encuestas. Sólo 143 de 4.200 AM no contestaron ninguna de ellas, y no fueron incluidos. De la encuesta PREVIAJE ( $n=802$ ), se encontró que $45,4 \%$ eran mayores de 70 años, 66,2\% eran mujeres; de aquellos que contestaron la encuesta POSTVIAJE ( $n=4.057), 42,2 \%$ eran mayores de 70 años y $69,1 \%$ eran mujeres. Al comparar ambos grupos, con respecto a edad y sexo, no encontramos diferencias por lo que estimamos que los resultados de la encuesta PREVIAJE son representativos de la población total de AM que viajó.

Los resultados de la encuesta PREVIAJE, se presentan en la Tabla 1. Respecto a previsión de salud: 60,5\% pertenecia al Fondo Nacional de Salud (FONASA) y 22,9\% a una Institución de Salud Previsional (Isapre). Educación: 20\% educación básica, $47 \%$ enseñanza media completa 0 parcial y $21 \%$ educación universitaria. Red de apoyo social: $15,8 \%$ vivía solo ( $82 \%$ son mujeres). En cuanto al ingreso económico: 7,7\% refería que su ingreso no le alcanzaba para cubrir necesidades básicas, a 43,1\% le alcanzaba para lo básico, sin darse gustos, mientras que $49,2 \%$ refiere no tener problemas económicos significativos, cifras que no se diferenciaron significativamente por sexo.

En relación con la priorización de temores a esa edad, lo más temido fue perder la salud, seguido por la dependencia mental, dependencia física, soledad y finalmente la pobreza. En cuanto a la priorización de valores, nuevamente el ítem más valorado fue tener buena salud, seguido por independencia física, compañía, dinero y por último tener un trabajo remunerado.

Evaluación geriátrica: en autopercepción de salud, más de la mitad de los AM que viajaron la consideró buena o excelente y sólo $2,2 \%$ la consideró mala. Sin embargo, al compararla con su salud del año anterior casi un tercio la consideró peor y sólo 22,1\% mejor. Déficit sensoriales: $25 \%$ refirió tener mala visión o tener problemas de audición a pesar de usar sus artefactos de apoyo (si es que los tenía). Casi 25\% consumía al menos cuatro medicamentos diariamente. Evaluación funcional: uno en diez de los AM que viajó tenía limitaciones para salir solo de casa y $14,3 \%$ requería ayuda para tomar sus medicamentos.

En tamizaje de depresión 21,2\% respondió afirmativamente a la pregunta única de depresión "ise siente frecuentemente triste o deprimido? y 16,2\% cumplió criterios de depresión, usando la Escala de Depresión Geriátrica acortada GDS-58.

Respecto a problemas geriátricos frecuentes: uno en diez refirió tener insomnio; 20,8\% haber tenido $\geq 1$ caída en los últimos tres meses, y uno en diez haber presentado incontinencia urinaria en los últimos tres meses. Hábitos: $12,5 \%$ era fumador activo.

Las patologías más frecuentes fueron: hipertensión arterial (48,1\%), osteoartrosis (26,8\%), osteoporosis $(23,8 \%)$, depresión $(16,7 \%)$, enfermedad cardíaca $(14,8 \%)$, diabetes mellitus $(13,9 \%)$ y enfermedad pulmonar crónica (EPOC o asma 3\%); 
Tabla 1. Encuesta PREVIAJE: C aracterísticas generales de Adultos M ayores que viajan $(n=802)$

\begin{tabular}{|c|c|}
\hline Carcaterísticas & $\%$ \\
\hline \multicolumn{2}{|l|}{ Evaluación social: } \\
\hline Vive solo & 15,8 \\
\hline Vive con familiares & 75,6 \\
\hline Sabe leer y escribir recados & 95,6 \\
\hline Hospitalización en últimos meses & 6,9 \\
\hline \multicolumn{2}{|l|}{ Autopercepción de su salud actual: } \\
\hline Excelente o buena & 59,4 \\
\hline Regular & 38,5 \\
\hline \multicolumn{2}{|c|}{ Autopercepción de su salud actual comparándola con la del año pasado: } \\
\hline Mejor 22,1 & \\
\hline Igual 50,3 & \\
\hline Peor $\quad 27,6$ & \\
\hline \multicolumn{2}{|l|}{ Evaluación geriátrica: } \\
\hline Tiene limitación funcional para salir solo de la casa & 9,5 \\
\hline Tiene limitación para mover muebles & 14,2 \\
\hline \multicolumn{2}{|l|}{ Medicamentos (consumo diario): } \\
\hline 0 ó 1 medicamento & 32,6 \\
\hline 2 ó 3 medicamentos & 43,7 \\
\hline 4 o más medicamentos & 23,6 \\
\hline Requiere ayuda en la toma de medicamentos & 14,3 \\
\hline Caídas $\geq 1$ (últimos 3 meses) & 20,8 \\
\hline Incontinencia urinaria (últimos 3 meses) & 11,7 \\
\hline Problemas de memoria mayor que la gente de su edad & 10,2 \\
\hline Insomnio frecuente & 14 \\
\hline \multicolumn{2}{|l|}{ Déficit sensorial: } \\
\hline Problemas de visión (incluso al usar lentes) & 25,9 \\
\hline Problemas de audición (incluso con audífonos) & 25,4 \\
\hline \multicolumn{2}{|l|}{ Tamizaje de depresión: } \\
\hline Pregunta única: ¿Se siente triste o deprimido? (sí) & 21,2 \\
\hline Escala de Depresión Geriátrica GDS-5 $(\geq 2 / 5)$ & 16,2 \\
\hline
\end{tabular}

osteoporosis, depresión, diabetes y cardiopatías fueron significativamente mayores en mujeres ( $p<0,05$ ). Los resultados de la encuesta de regreso 0 POSTVIAJE, se presentan en la Tabla 2. Respecto al impacto biomédico de los viajes, destaca una franca mejoría en casi todos los ítemes preguntados, que superan $73 \%$ en los ítemes de ánimo, sociabilidad y bienestar general, con menos de $0,6 \%$ de deterioro en cada uno de ellos; seguidos por los ítemes insomnio, apetito y molestias articulares, con más de $55 \%$ de los AM refiriendo mejoría. Al realizar un análisis por subgrupos según edad, pudimos comprobar que el impacto de los viajes no dependió de la edad, salvo en lo que se refiere a molestias articulares y ánimo en
Tabla 2. Encuesta PO ST VIAJE: Impacto biomédico en adultos mayores que viajaron $(n=4.057)$

\begin{tabular}{|llll|}
\hline & Peor & $\begin{array}{c}\text { Igual } \\
\text { (\%) }\end{array}$ & Mejor \\
\hline Ánimo & 0,6 & 14 & 85,4 \\
Sociabilidad & 0,6 & 20,7 & 78,7 \\
Bienestar general & 0,5 & 26,3 & 73,2 \\
Insomnio & 2,4 & 38,9 & 58,7 \\
Molestias articulares & 3,1 & 41,1 & 55,8 \\
Apetito & 1,0 & 41 & 58 \\
Memoria & 1,2 & 54,4 & 44,4 \\
Digestión & 8,5 & 49,4 & 42,1 \\
\hline
\end{tabular}


que los $<75$ años mostraron significativamente más alivio $(\mathrm{p}<0,05)$; sin embargo, sí dependió del género del sujeto, ya que se encontró que las mujeres, en comparación con los hombres, experimentaron cifras estadísticamente significativas ( $\mathrm{p}$ $<0,05)$, de mayor mejoría en todos los campos excepto en ánimo y apetito.

\section{DisCUSIÓN}

El aumento sostenido de AM en Chile en la última década, genera necesidades que debemos afrontar, ya que ellos son los nuevos usuarios de los servicios médico-sociales ${ }^{2,3}$. Este hecho es una realidad en los países más desarrollados, por lo que se han impulsado diferentes iniciativas, fomentando la integración social del AM y potenciando sus posibilidades de participación en distintas actividades laborales y recreativas ${ }^{4,5}$. Esto con un doble propósito: beneficiar a los AM y ser un aporte no menos importante al desarrollo económico del país. Así nacieron los programas subvencionados de fomento de viajes para los AM, que fueron desarrollados en la década 199099 en Europa y en el año 2001 se iniciaron en Chile con el programa "Vacaciones de Tercera Edad en Baja Temporada" de SERNATUR. Sin duda, iniciativas como ésta, que utilizan recursos en un país en vías de desarrollo, requieren una evaluación cuidadosa de su posible impacto. En este trabajo describimos el perfil de las personas que participaron en el programa y cuál fue el impacto biomédico del viaje, desde un punto de vista de bienestar general y en algunos aspectos de salud.

Los resultados nos describen el perfil del AM que viajó, destacándose que dos de cada tres son mujeres; uno de cada cinco tiene 75 años o más y uno de cada seis vive solo. Estas cifras revelan que el fenómeno del envejecimiento en Chile comparte características ya reportadas en los países más desarrollados, como una mayor esperanza de vida de las mujeres, que supera en 3,8 años a la del hombre ${ }^{7}$, con un mayor porcentaje de solteras y viudas (27,4\% mujeres v/s $8,3 \%$ de los hombres) ${ }^{7,8}$. Desde el punto de vista educacional, en general, este grupo muestra niveles algo superiores al resto de la población chilena mayor de 60 años $^{7}$, ya que a nivel nacional $87 \%$ tiene un alfabetismo adecuado versus el $96 \%$ de los AM que viajó reporta saber leer o escribir un recado.

Del análisis de las características del grupo, destaca que los AM que viajan priorizan su salud como el elemento más preciado y el que más temen perder. Este grupo coloca en segundo lugar de valoración el tener compañía y en tercero los aspectos económicos.

Con respecto a la salud, encontramos que la mayoría la considera buena o excelente (59\%), aun cuando refieren similares prevalencias de enfermedades crónicas que lo encontrado en Chile por el estudio SABE de la OPS y en otros estudios, al menos en lo que se refiere a hipertensión arterial, diabetes mellitus y osteoartrosis 7,8 . Destaca la alta prevalencia de depresión, cifras que aparecen tanto como autorreporte de patología diagnosticada por médico (16,7\%), como en la pregunta única "ise siente usted frecuentemente triste o deprimido?' (21\%), como también utilizando una escala validada de Depresión Geriátrica GDS-56 en que $16 \%$ de los AM cumple criterios de depresión. Estos resultados son similares (24\%) a los obtenidos en el estudio SABE que fue realizado en una muestra representativa de AM que viven en sus hogares en la Región Metropolitana ${ }^{7}$ y revelan la importancia de esta patología en nuestro país, incluso en grupos como éste en que uno podría esperar prevalencias menores, ya que los deprimidos tienden a participar menos de actividades sociales y recreativas.

En relación con aspectos de la valoración geriátrica, destaca que el grupo de AM que viaja mostró menor prevalencia de incontinencia urinaria (12\%) y caídas (21\%) que lo anteriormente reportado en el estudio $\mathrm{SABE}^{7}, 25 \%$ y $35 \%$, respectivamente. Sin embargo, es un problema importante la pérdida sensorial, 25\% de limitación en visión o audición, a pesar de usar artefactos de apoyo cuando los tienen. Esto altera significativamente la funcionalidad del AM y los expone a mayores riesgos de fragilidad y dependencia, al igual que la polifarmacia que afecta también a un cuarto del grupo que viaja.

El análisis de los resultados POSTVIAJE nos revela el positivo impacto biomédico del viaje en distintos aspectos subjetivos del bienestar general y la salud tanto física como mental. Estos resultados son categóricos en el beneficio que los viajes tienen en los $\mathrm{AM}$, ya que todos los ítemes 
analizados mostraron grandes mejorías, destacando lo ocurrido con el ánimo, que era una patología prevalente y $86 \%$ notó mejoría. El porcentaje relativo que sintió empeoramiento de sus molestias fue insignificante y las categorías que menos se modificaron fueron la memoria y la digestión, hechos esperables. Existen pocas experiencias descritas en la literatura sobre el efecto biomédico que producen los viajes en el adulto mayor. Hay una que los recomienda desde el punto de vista general ${ }^{9}$ y encontramos una experiencia alemana, publicada hace más de dos décadas, en que pacientes con patología psiquiátrica fueron sometidos a un "viaje terapéutico" con buenos resultados ${ }^{10}$.

Como aspectos a considerar en la validez de los resultados de este estudio, creemos que, al ser un estudio prospectivo, donde las encuestas fueron aplicadas en forma no seleccionada a todos

\section{REFERENCIAS}

1. Instituto Nacional de Estadística (INE). Censo de población y vivienda, Chile. Resultados Generales. 2002.

2. Marín PP. La situación del adulto mayor en Chile (artículo especial). Rev Méd Chile 1998; 125: 120712.

3. Marín PP. Demografía y epidemiología del envejecimiento en Chile. En: Marín PP, Manual de Geriatría y Gerontología. Santiago: Ediciones Universidad Católica de Chile 2002; 47-54.

4. Report of the World Assembly on Ageing, Vienna, 1982 (United Nations publication, Sales № E.82.I.16).

5. Segunda Asamblea Mundial sobre el Envejecimiento (Madrid 2002) http://www.onu.org/Agen$\mathrm{da} /$ conferencias/ envejecimiento.

6. Hoyl T, Valenzuela E, Marín PP. Depresión en el Adulto Mayor: Evaluación Preliminar de la Efectividad como Instrumento de Tamizaje de la Versión de 5 ítemes de la Escala de Depresión Geriátrica. Rev Méd Chile 2000; 128: 1199-204. los participantes, le da valor a los resultados. La diferencia entre las encuestas respondidas de ida versus las de regreso podría explicarse porque las respuestas eran voluntarias y la encuesta de regreso era mucho más corta y tal vez los participantes tenían una mejor disposición para responder después de sus vacaciones. Sin embargo, las características por sexo y edad eran comparables y los resultados no debieron verse afectados porque eran preguntas independientes. En el futuro, sería interesante evaluar con un seguimiento a lango plazo, el tiempo de permanencia de los cambios positivos observados después del viaje.

Consideramos que el presente estudio aporta evidencias que avalan la importancia de fomentar este tipo de iniciativas de recreación para todos los AM, para así promover la integración social y mejorar la calidad de vida.

7. Perfil del Adulto Mayor en Chile. Desarrollando respuestas integradas de sistemas de cuidados de salud para una población de rápido envejecimiento (INTRA). Publicación del MINSAL, OPSOMS, 2002.

8. Análisis de la VIII Encuesta CASEN: Situación de Salud 2000. Ministerio de Planificación y Cooperación, Gobierno de Chile. Santiago de Chile, Agosto 2001 (http://www.mideplan.cl/estudios/ informesalud2000.pdf).

9. Strause-Blasche G, Ekmekcioglu C, Marktl W. Does vacation enable recuperation? Changes in wellbeing associated with time away from work. Occupational Medicine 2000; 50: 167-72.

10. Stephan R, Ronnecke R, Gutzmann H. Therapeutic travel with patients in a gerontopsychiatric polyclinic. Zeitschrift fur Gerontolologie 1981; 14: 75-82.

\section{Agradecimientos}

Los autores agradecen la colaboración de los encargados y monitores de IberoJet en entregar y recoger las encuestas de evaluación; y a la Fundación Adulto Mayor-FAM de España por su constante estímulo en la realización del estudio. 